\title{
Aspectos da Sexualidade e Gravidez em Adolescentes com Artrite Idiopática Juvenil (AIJ) $)^{(*)}$
}

\section{Sexuality Aspects and Presnancy of Adolescents with Juvenile Idiopathic Arthritis (JA)}

\author{
Clovis Artur Almeida Silva ${ }^{(1)}$, Ricardo Maisse Suehiro(2), Marta Miranda Leal ${ }^{(3)}$, Bernadete Lourdes Liphaus ${ }^{(4)}$, \\ Lucia Maria M. A. Campos ${ }^{(4)}$, Venina Viana de Barros ${ }^{(5)}$, Marcelo Zugaib ${ }^{(6)}$
}

\section{RESUMO}

O presente trabalho tem como objetivo descrever aspectos da sexualidade, gravidez e pós-parto de três adolescentes com artrite idiopática juvenil (AIJ). No período entre 1983 e 2004, 4.638 pacientes foram acompanhados na Unidade de Reumatologia Pediátrica do Departamento de Pediatria da FMUSP, entre os quais 537 (11,5\%) apresentaram o diagnóstico de AIJ (critérios do ILAR). Entre os pacientes com AIJ, três engravidaram durante o seguimento ambulatorial. A idade da primeira atividade sexual variou de 16 a 18 anos. A paciente 1 apresentou uma gestação gemelar a termo, permanecendo em atividade da doença durante toda a gravidez, em uso de $15 \mathrm{mg} /$ dia de prednisona. As pacientes 2 e 3 encontravamse em remissão da doença, sem uso de medicamentos, apresentando gestação a termo sem intercorrências. A paciente 2, porém, evoluiu com recidiva da doença um ano após o parto. Todos os recémnascidos foram adequados para idade gestacional e evoluíram adequadamente no período neonatal. Apenas a paciente 1 necessitou de prednisona, naproxeno e cloroquina na amamentação. $\mathrm{O}$ aumento da gravidez na adolescência é uma realidade nos serviços de reumatologia pediátrica, o que impõe novos debates sobre os aspectos da sexualidade e contracepção nessa população.

Palavras-chave: artrite idiopática juvenil, adolescência, sexualidade, gravidez.

\begin{abstract}
The objective of the present study is to describe the sexuality aspects, pregnancy and postpartum in three adolescents with juvenile idiopathic arthritis (JIA). From 1983 to 2004, 4,638 patients were followed at the Pediatric Rheumatology Unit of the Pediatric Department of FMUSP, of which 537 (11.5\%) were diagnosed with JIA (ILAR criteria) and three of these patients became pregnant during the follow-up period. The age at their first sexual intercourse ranged from 16 to 18 years old. Patient 1 presented a twin pregnancy, with active disease throughout pregnancy, and was on $15 \mathrm{mg}$ of prednisone per day. Patients 2 and 3 were at disease remission, with no drug treatment, and had full-term pregnancies free of complications; patient 2, however, had a disease flare-up one year after the delivery. All newborns were adequate for gestational age and developed no complications during the neonatal period. Only patient 1 required prednisone, naproxen e chloroquine during breastfeeding. The increased frequency of pregnancy in adolescents is a reality in pediatric rheumatology centers, and it shows the importance of more debate concerning sexuality aspects and contraception in this population.
\end{abstract}

Keywords: juvenile idiopathic arthritis, adolescence, sexuality, pregnancy.

\footnotetext{
Trabalho realizado nas Unidades de Reumatologia Pediátrica e de Adolescente do Departamento de Pediatria e Disciplina de Obstetrícia da Faculdade de Medicina da Universidade de São Paulo (FMUSP), São Paulo, SP, Brasil. Recebido em 15/02/2005. Aprovado, após revisão, em 19/03/2005.

1. Doutor em Medicina pela FMUSP. Responsável pela Unidade de Reumatologia Pediátrica do Departamento de Pediatria da FMUSP

2. Médico da Complementação Especializada da Unidade de Reumatologia Pediátrica do Departamento de Pediatria da FMUSP.

3. Mestre em Medicina pela FMUSP. Médica assistente da Unidade de Adolescente do Departamento de Pediatria da FMUSP.

4. Mestre em Medicina pela FMUSP. Médica assistente da Unidade de Reumatologia Pediátrica do Departamento de Pediatria da FMUSP.

5. Doutor em Medicina pela FMUSP. Médica assistente do Setor de Colagenoses da Disciplina de Obstetrícia da FMUSP.

6. Professor titular da Disciplina de Obstetrícia da FMUSP.

Endereço para correspondência: Dr. Clovis Artur Almeida da Silva. R. Senador Cesar Lacerda Vergueiro, 494/82, Vila Madalena, CEP 05435-010. São Paulo, SP, Brasil. Tel. (11) 3069-8675; fax (11) 3069-8503; e-mail: clovisaas@icr.hcnet.usp.br
} 


\section{INTRODUÇÃO}

Atualmente, com a melhora do prognóstico e da qualidade de vida de pacientes com artrite idiopática juvenil (AIJ), os ambulatórios de reumatologia pediátrica têm tido que lidar com uma população cada vez maior de adolescentes e, portanto, com questões próprias da adolescência, entre elas: o desenvolvimento da sexualidade, o início cada vez mais precoce da atividade sexual e suas possíveis consequiências como doenças sexualmente transmissíveis (DST) e gravidez.

Alguns estudos avaliaram aspectos da sexualidade em pacientes com AIJ. Britto et al.(1) estudaram 178 adolescentes com doenças reumáticas pediátricas, $69 \%$ com AIJ e $8 \%$ com lúpus eritematoso sistêmico juvenil (LESJ). Das 52 adolescentes do sexo feminino, 31 (60\%) eram sexualmente ativas. Onze (41\%) das 27 adolescentes sexualmente ativas questionadas sobre anticoncepção não utilizaram métodos contraceptivos. Packman e Hall( ${ }^{(2)}$ avaliaram a atividade sexual em 246 adultos ingleses com AIJ, sendo 176 (72\%) do sexo feminino. A média de idade da primeira relação sexual foi 19,3 $\pm 3,9$ anos, sendo que 37,6\% delas haviam iniciado vida sexual antes dos 18 anos de idade.

Um estudo realizado na população brasileira demonstrou que $42,5 \%$ das mulheres iniciam sua atividade sexual antes dos 18 anos de idade e, em 1996, cerca de um quarto dos partos realizados no país foram em adolescentes ${ }^{(3)}$. As características da adolescência envolvendo crescimento e desenvolvimento físico e emocional incompletos, mudanças no processo de socialização, desenvolvimento da identidade sexual e da identidade adulta conferem à gestação adolescente uma complexidade muito grande, acarretando agravos físicos e psicossociais para mães e filhos ${ }^{(4)}$. Além disso, observa-se retardo do início do acompanhamento pré-natal e menor freqüência às consultas entre as adolescentes grávidas, provavelmente pela falta de maturidade necessária nessa situação.

$\mathrm{Na}$ gestação de uma jovem com AIJ, esses fatores agravantes adquirem dimensão ainda maior, uma vez que pode haver reativação da doença, com conseqüências maternas, fetais e no recém-nascido (RN). Por sua vez, as limitações nas pacientes com AIJ, tais como redução da abdução da articulação coxofemoral ou baixa estatura, podem constituir indicação de parto cesariano em até $79 \%$ dessas pacientes ${ }^{(2)}$.

O presente trabalho tem como objetivo avaliar aspectos da sexualidade, gravidez e pós-parto em adolescentes com AIJ. No período entre 1983 e 2004, 4.638 pacientes foram acompanhados na Unidade de Reumatologia Pediátrica do Departamento de Pediatria da FMUSP, entre os quais
$537(11,5 \%)$ apresentaram o diagnóstico de AIJ (critérios do ILAR $)^{(5)}$. Entre os pacientes com AIJ, três engravidaram durante o seguimento ambulatorial.

\section{RELATO DE CASOS}

\section{CASO 1}

Adolescente atualmente com 19 anos e 6 meses, branca, natural e procedente de São Paulo, apresentando o diagnóstico de AIJ desde os cinco anos de idade. A forma de início da AIJ foi poliarticular, FAN positivo, FR positivo e evolução poliarticular (comprometimento dos joelhos, tornozelos e cotovelos). Aos 11 anos, apresentou iridociclite anterior silenciosa ao exame de lâmpada de fenda, com remissão após tratamento com colírios de dexametasona e atropina. Durante o seguimento, necessitou de vários tratamentos para o controle da doença, com as seguintes doses cumulativas: indometacina $(27,9 \mathrm{~g})$, ibuprofeno $(450 \mathrm{~g})$, naproxeno $(1545 \mathrm{~g})$, metotrexato $(6,48 \mathrm{~g})$, prednisona $(15,07 \mathrm{~g})$ e hexacetonide de triamcinolona intra-articular (320 mg, utilizado em doses de $40 \mathrm{mg}$, uma vez por ano, em quatro vezes para cada joelho). A idade da menarca foi de 11 anos e 10 meses. Aos 12 anos e 3 meses, os ciclos menstruais eram normais e associados à dismenorréia. A primeira atividade sexual foi com 17 anos e 10 meses, com freqüência de uma relação sexual por semana. A paciente relatava motivação sexual e orgasmo normal, porém negava masturbação. Apesar das orientações prévias sobre contracepção, o casal fazia uso irregular de método contraceptivo de barreira (preservativo masculino). Aos 18 anos e 4 meses por conta própria suspendeu todas as medicações em uso: prednisona $5 \mathrm{mg} /$ dia, naproxeno $500 \mathrm{mg} /$ dia e metotrexato $20 \mathrm{mg} / \mathrm{semana}$. Aos 18 anos e 7 meses retornou com gravidez de 12 semanas. A gravidez foi não planejada, porém desejada, recebendo o apoio da família e de seu parceiro. Nesse momento, a doença encontrava-se ativa (artrites no cotovelo esquerdo, joelhos e tornozelo direito), VHS $30 \mathrm{~mm} / 1^{\text {a }}$ hora, PCR $<3,2 \mathrm{mg} / \mathrm{dl}$, Childhood Health Assessment Questionnaire (CHAQ) ${ }^{(6)}$ 0,125 e classe funcional II do American College of R heumatology (ACR) ${ }^{(7)}$, sendo mantida apenas a prednisona $15 \mathrm{mg} /$ dia durante toda a gestação. No início da gravidez, os seguintes exames laboratoriais foram realizados: hemoglobina de $10,8 \mathrm{~g} / \mathrm{dl}$, contagem de plaquetas de $323.000 / \mathrm{mm}^{3}$ e de leucócitos de 2.390/ $\mathrm{mm}^{3}$ (56\% neutrófilos, 36\% linfócitos, 7\% monócitos). A urinálise foi normal. As sorologias para sífilis, HIV, toxoplasmose, Chagas e hepatite B e C foram negativas. 
O pré-natal foi realizado com 12 consultas e evoluiu sem intercorrências, porém a paciente manteve atividade clínica (artrite nos joelhos) durante todo o período gestacional e no puerpério. A gravidez foi gemelar, com idade gestacional de 37 semanas e o parto foi cesariano. Os RN, ambos do sexo feminino, foram de termo (RNT) e adequados para a idade gestacional (AIG). A primeira gemelar apresentou Apgar 7/8 (no primeiro e quinto minutos, respectivamente), peso de $2.490 \mathrm{~g}$, estatura de $44 \mathrm{~cm}$ e perímetro cefálico de $32,5 \mathrm{~cm}$. A segunda gemelar apresentou Apgar $7 / 8$, peso de $2.530 \mathrm{~g}$, estatura de $44 \mathrm{~cm}$ e perímetro cefálico de $34 \mathrm{~cm}$. Ambas evoluíram com icterícia fisiológica, sem outras intercorrências no período neonatal. A paciente atualmente encontra-se amamentando, em uso de naproxeno $500 \mathrm{mg} /$ dia, prednisona $15 \mathrm{mg} /$ dia e difosfato de cloroquina $250 \mathrm{mg} /$ dia.

\section{CASO 2}

Jovem com idade atual de 20 anos e 11 meses, parda, natural e procedente de São Paulo, apresentando o diagnóstico de AIJ desde os seis anos e sete meses de idade. A forma de início da AIJ foi oligoarticular persistente (comprometimento dos punhos e joelhos), FAN negativo e FR negativo. O exame oftalmológico com lâmpada de fenda foi normal. Durante o seguimento necessitou de vários tratamentos para o controle da doença, com as seguintes doses cumulativas: indometacina $(80,2 \mathrm{~g})$, naproxeno $(408,4 \mathrm{~g})$ e sulfasalazina (1950g). A idade da menarca foi de 14 anos. Aos 14 anos e 6 meses, os ciclos menstruais eram normais e associados à dismenorréia. A primeira atividade sexual foi com 16 anos, com frequiência de uma relação sexual por mês. A paciente relatava motivação sexual e orgasmo normal, porém negava masturbação. Apesar das orientações prévias sobre contracepção, o casal fazia uso irregular de método contraceptivo de barreira (preservativo masculino). Aos 17 anos e 5 meses, suspendeu por conta própria as medicações em uso (indometacina e sulfasalazina) por suspeita de gravidez. Aos 17 anos e 6 meses retornou em consulta, grávida de nove semanas, sendo mantida sem medicação. Nesse momento, a doença encontrava-se em remissão, classe funcional I do $\mathrm{ACR}^{(7)}$, sem limitações articulares, assim permanecendo durante toda a gestação e puerpério. A gravidez não foi planejada, porém desejada, recebendo o apoio da família e de seu parceiro. Apresentava na época os seguintes exames laboratoriais: hemoglobina de $12,8 \mathrm{~g} / \mathrm{dl}$, contagem de plaquetas de $162.000 / \mathrm{mm}^{3}$ e de leucócitos de $6.800 / \mathrm{mm}^{3}$ (67\% neutrófilos, $22 \%$ linfócitos, $10 \%$ monócitos), VHS $9 \mathrm{~mm} / 1^{a}$ hora e $\mathrm{PCR}<3,2 \mathrm{mg} / \mathrm{dl}$. A urinálise foi normal. As sorologias para sífilis, HIV, toxoplasmose, doença de Chagas e hepatites B e C foram negativas. O pré-natal foi realizado com dez consultas e evoluiu sem intercorrências. A gestação foi de 37 semanas, com parto vaginal e sem intercorrências. O RNT-AIG foi do sexo masculino, peso de 2610g, Apgar 9/10 (no primeiro e quinto minutos, respectivamente) e recebeu alta hospitalar com 24 horas de nascimento. Após um ano e 2 meses do parto, aos 19 anos e 2 meses, a paciente apresentou reativação da doença, sendo reintroduzido naproxeno, o qual foi mantido por seis meses.

\section{CASO 3}

Jovem com idade atual de 20 anos e 8 meses, negra, natural e procedente de São Paulo, apresentando o diagnóstico de AIJ desde os 10 anos de idade. A forma de início da AIJ foi oligoarticular estendida (comprometimento dos punhos, interfalangeanas proximais, joelhos e tornozelos), FAN negativo e FR negativo. Durante o seguimento necessitou de tratamento para o controle da doença, com as seguintes doses cumulativas: naproxeno $(1.190 \mathrm{~g})$ e metotrexato $(2,96 \mathrm{~g})$. Aos 18 anos e 6 meses de idade foi também submetida à infiltração de com hexacetonide de triamcinolona com 40mg em cada joelho. A idade da menarca foi aos 12 anos. Aos 12 anos e 6 meses, os seus ciclos menstruais eram regulares, associados à dismenorréia. A primeira relação sexual foi aos 18 anos, com uma freqüência de duas relações sexuais por semana. A paciente relatava motivação sexual e orgasmo normal, porém referia dispareunia em algumas relações. Negava a prática de masturbação. Seu parceiro usava preservativo, porém de forma irregular. Aos 19 anos e 10 meses estava grávida de 13 semanas, sendo então suspenso o naproxeno. Nesse momento, a doença encontrava-se inativa, sem limitações articulares, classe funcional I do $\mathrm{ACR}^{(7)}$, assim permanecendo durante toda a gestação e pós-parto. A gravidez foi não planejada, porém desejada, recebendo o apoio de seu parceiro. Os seguintes exames laboratoriais foram realizados no início da gestação: hemoglobina de 10,4g/dl, contagem de plaquetas de 336.000/ $\mathrm{mm}^{3}$ e de leucócitos de $7.010 / \mathrm{mm}^{3}$ (51\% neutrófilos, 37\% linfócitos, $11 \%$ monócitos), VHS $26 \mathrm{~mm} / 1^{\text {a }}$ hora, PCR $<3,2 \mathrm{mg} / \mathrm{dl}$. A urinálise foi normal. As sorologias para sífilis, HIV, toxoplasmose, doença de Chagas e hepatites B e C foram negativas. O pré-natal foi realizado com dez consultas e evoluiu sem intercorrências. A gestação foi de 38 semanas, com parto vaginal e sem intercorrências. O RNT-AIG era do sexo feminino, Apgar 9/10 (no primeiro e quinto minuto respectivamente), peso $2.860 \mathrm{~g}$, estatura de $46 \mathrm{~cm}$, 
perímetro cefálico de $34 \mathrm{~cm}$, recebendo alta hospitalar 24 horas após o nascimento. Atualmente, a paciente está em remissão da doença.

\section{DISCUSSÃo}

As pacientes com AIJ tornam-se adolescentes com vida sexual ativa com possibilidade de constituir família. A escassez de publicações, em literatura médica, sobre a sexualidade e função gonadal das adolescentes com AIJ estimulou a realização deste estudo.

A gravidez na adolescência e o início precoce da atividade sexual são problemas universais que atingem tanto países desenvolvidos quanto subdesenvolvidos, embora principalmente estes últimos ${ }^{(8)}$. No Brasil, em 1997, mais de 700.000 partos $\left(26 \%\right.$ do total) foram em mulheres adolescentes ${ }^{(9)}$.

Em nosso Hospital Universitário e em nossa Unidade de Reumatologia Pediátrica tem sido observado um aumento da frequiência de gravidez em adolescentes portadoras de doenças crônicas, refletindo o que está acontecendo na sociedade como um todo. Trabalho realizado com 43 adolescentes com LESJ, seguidas em nosso serviço e Disciplina de Obstetrícia da FMUSP durante os anos de 1999 e 2000, demonstrou a ocorrência de gravidez em seis $(14 \%)$ dessas pacientes ${ }^{(10)}$.

As taxas de fertilidade na AIJ podem estar diminuídas ${ }^{(11)}$. Nessa doença, a redução da função ovariana pode ocorrer pela própria atividade da doença crônica e/ou uso dos imunossupressores. A azatioprina e o metotrexato, habitualmente, não provocam infertilidade, que é evidenciada com o uso de pulsoterapia com ciclofosfamida e clorambucil ${ }^{(12)}$. Packham e Hall ${ }^{(1)}$ avaliaram 177 pacientes adultas com AIJ, com idade média de 35,4 anos (19 a 78 anos) e duração média da doença de 28,7 anos. Dessas, seis $(3,4 \%)$ apresentaram falência ovariana prematura idiopática (com amenorréia mantida) comparada a uma incidência de $1 \%$ na população geral. No entanto, Silva et al..$^{(13)}$ evidenciaram que, em adolescentes com LESJ (mediana de idade de 16 anos), a reserva folicular ovariana foi normal apesar da atividade da doença ou uso de medicamentos.

A AIJ pode apresentar-se ou recidivar antes, durante ou após a gravidez. Neste estudo, nas três pacientes a doença foi prévia à gestação.

A evolução da doença durante a gravidez e no puerpério ainda não está bem estabelecida, com poucos casos relatados, geralmente em adultas com AIJ. Alguns estudos ${ }^{(14,15)}$ mostram uma melhora da atividade e até mesmo remissão durante a gestação, podendo ocorrer, entretanto, recidivas no puerpério (após 3 a 6 meses do parto) e na amamentação. A AIJ na paciente 1 manteve-se ativa durante toda a gestação, puerpério e amamentação. Por sua vez, as pacientes 2 e 3 estavam em remissão da doença no momento da gestação e puerpério, sendo que a paciente 2 evoluiu com recidiva um ano após o parto.

O prognóstico fetal está relacionado à atividade da doença materna, podendo ocorrer abortamento, retardo do crescimento intra-uterino, prematuridade e óbito neonatal. Østensen et al. ${ }^{(14)}$ mostraram que o aborto espontâneo ocorreu com maior frequiência em pacientes com AIJ em relação aos controles. No estudo de Packham e Hall(2), $33 \%$ de todas as gestações de mulheres com AIJ evoluíram para abortamento espontâneo. Na presente casuística, todos os recém-nascidos foram a termo e evoluíram adequadamente no período neonatal. O parto foi cesariano apenas na paciente 1 , por se tratar de gestação gemelar em paciente primigesta. Em nenhum dos três casos houve envolvimento da articulação coxofemoral, o que habitualmente é indicação de parto cesariano.

Nenhuma de nossas pacientes encontrava-se em uso de imunossupressores durante a gestação e apenas a paciente 1 fez uso de prednisona durante toda a gravidez. Corticosteróides, em altas doses, podem causar fenda palatina em modelos experimentais com animais e baixo peso ao nascimento em seres humanos. No entanto, não há evidências de que a prednisona ou pulsoterapia com metilprednisolona sejam teratogênicas em humanos, sendo consideradas categoria de risco B, segundo a Food and Drug Administration ${ }^{(16)}$. Após o parto, como a paciente 1 mantinha atividade importante da doença, foram associados naproxeno e difosfato de cloroquina. A Associação Americana de Pediatria considera naproxeno, prednisona e anti-malárico como medicamentos compatíveis com amamentação ${ }^{(17)}$.

Os dados apresentados chamam a atenção para a ocorrência de atividade sexual e gravidez em adolescentes portadoras de AIJ. Quando se compreende que o desenvolvimento da sexualidade envolve questões como socialização, maturidade física, imagem corporal, auto-estima, aspirações referentes ao futuro e a construção da própria identidade, consegue-se entender como a doença interfere nesse processo e é um determinante de risco ${ }^{(8)}$.

Nesse sentido, o médico especialista ocupa um papel fundamental, pois, muitas vezes, é a única referência de profissional de saúde na orientação sexual das pacientes. Dessa forma, o reumatologista pediátrico deve reservar, durante a consulta, um espaço que lhe permita abordar e discutir aspectos da sexualidade, orientar sobre os métodos 
contraceptivos e alertar quanto aos riscos da gestação e prevenção de doenças sexualmente transmissíveis.

O Colégio Americano de Reumatologia preconiza que os reumatologistas devem se responsabilizar pelos cuidados preventivos dos pacientes com patologias reumáticas crônicas, incluindo abordagem dos aspectos da sexualidade e contracepção ${ }^{(18)}$. Os métodos de barreira, como diafragma e preservativo masculino ou feminino, são considerados seguros e eficazes em adolescentes com AIJ, devendo ser utilizados em todas as relações sexuais. Os preservativos devem ser sempre

\section{REFERÊNCIAS}

1. Britto MT, Rosenthal SL, Taylor J, Passo MH: Improving rheumatologists screening for alcohol use and sexual activity. Arch Pediatr Adolesc Med 154: 478-83, 2000.

2. Packham JC, Hall MA: Long-term follow-up of 246 adults with juvenile idiopathic arthritis: social function, relationships and sexual activity. Rheumatology 41: 1440-3, 2002.

3. Badiani R, Quental I, Santos EM: DST/AIDS e a pesquisa nacional sobre demografia e saúde: uma análise do nível de conhecimento e comportamento de vulnerabilização. Rio de Janeiro, BEMFAM/ DHS, 1997.

4. Sharma AK, Verma K, Khatri S, Kannan AT: Pregnancy in adolescents: Study of Risks and Outcome in Eastern Nepal. Indian Pediatr 38: 1405-9, 2001.

5. Petty RE, Southwood TR, Manners P, et al: International League of Associations for Rheumatology classification of juvenile idiopathic arthritis: second revision, Edmonton, 2001. J Rheumatol 31: 390-2, 2004.

6. Machado CS, Ruperto N, Silva CH, et al: Paediatric Rheumatology International Trials Organization. The Brazilian version of the Childhood Health Assessment Questionnaire (CHAQ) and the Child Health Questionnaire (CHQ). Clin Exp Rheumatol 19: S25-30, 2001.

7. Hochberg MC, Chang RW, Dwosh I, et al: The American College of Rheumatology 1991 revised criteria for the classification of global functional status in rheumatoid arthritis. Arthritis Rheum 35: 498, 1992.

8. Saito MI: Sex education in school: preventing unwanted pregnancy in adolescents. Inter J Gynecol Obstetr 63: 157-60, 1998.

9. Serviço de Assistência à Saúde do Adolescente do Ministério da Saúde (SASAD): Prevenção intersetorial da gravidez na adolescência. Documento preliminar. São Paulo, Brasil, 1997. utilizados pelos adolescentes, mesmo com a utilização conjunta de outros métodos contraceptivos, como preventivo para DST. Os contraceptivos hormonais (combinados ou não) não estão contra-indicados nas portadoras de $\mathrm{AJJ}^{(19)}$ e podem ser indicados nas pacientes com atividade sexual freqüente e/ou naquelas que solicitem tais métodos.

O aumento da gravidez na adolescência é também uma realidade nos ambulatórios de reumatologia pediátrica, o que impõe novas discussões sobre os aspectos da sexualidade e contracepção nos pacientes com AIJ.

10. Silva CA, Leal MM, Campos LM, et al: Aspectos da sexualidade e gravidez de adolescentes e adultos jovens com lúpus eritematoso sistêmico juvenil (LES). Rev Bras Reumatol 41: 213-9, 2001.

11. Packham JC, Hall MA: Premature ovarian failure in women with juvenile idiopathic arthritis (JIA). Clin Exp Rheumatol 21: 347-50, 2003.

12. Clements PJ, Davis J: Cytotoxic drugs: their clinical application to the rheumatic diseases. Semin Arthritis Rheum 15: 231-54, 1986.

13. Silva CA, Leal MM, Leone C, et al: Gonadal function in adolescents and young women with juvenile systemic lupus erythematosus. Lupus 11: 419-25, 2002.

14. Ostensen M, Almberg K, Koksvik HS: Sex, reproduction, and gynecological disease in young adults with a history of juvenile chronic arthritis. J Rheumatol 27: 1783-7, 2000.

15. Musiej-Nowakowska E, Ploski R: Pregnancy and early onset pauciarticular chronic arthritis. Ann Rheum Dis 58: 475-80, 1999.

16. Janssen NM, Genta MS: The effects of immunosuppressive and antiinflammatory medications on fertility, pregnancy, and lactation. Arch Intern Med 160: 610-9, 2000.

17. Committee on Drugs, American Academy of Pediatrics. The transfer of drug and other chemical into human milk. Pediatrics 93: 137-50, 1994.

18. American College of Rheumatology. The rheumatologist as principal care physician: In: ACR's Annual Scientific Meeting, Atlanta, 1998. p. 1-5.

19. Hannaford PC, Kay CR, Hirsch S: Oral contraceptives and rheumatoid arthritis: new data from the Royal College of General Practitioners' oral contraception study. Ann Rheum Dis 49: 744-6, 1990. 